EPJ manuscript No.

(will be inserted by the editor)

\title{
Uncertainty principle for control of ensembles of oscillators driven by common noise
}

\author{
Denis S. Goldobin ${ }^{1,2, a}$ \\ 1 Institute of Continuous Media Mechanics, UB RAS, Perm 614013, Russia \\ 2 Department of Mathematics, University of Leicester, Leicester LE1 7RH, UK, EU
}

\begin{abstract}
We discuss control techniques for noisy self-sustained oscillators with a focus on reliability, stability of the response to noisy driving, and oscillation coherence understood in the sense of constancy of oscillation frequency. For any kind of linear feedback control-single and recursive delay feedback, linear frequency filter, etc.- the phase diffusion constant, quantifying coherence, and the Lyapunov exponent, quantifying reliability, can be efficiently controlled but their ratio remains constant. Thus, an "uncertainty principle" can be formulated: the loss of reliability occurs when coherence is enhanced and, vice versa, coherence is weakened when reliability is enhanced. Treatment of this principle for ensembles of oscillators synchronized by common noise or global coupling reveals a substantial difference between the cases of slightly non-identical oscillators and identical ones with intrinsic noise.
\end{abstract}

\section{Introduction}

Collective phenomena in ensembles of dynamical systems can manifest complex behavior and self-organization, which are not possible for unitary systems with arbitrary level of complexity. For biological systems, they extend from the simplest collective dynamics of bacteria [1] or higher organisms [2] to the activity of neural tissue [34]. Similarly, in technology, essentially collective phenomena vary from plain synchronization effects $5|6| 7$ to the phenomena laying in the basis of operation of artificial neural networks 38. Although these phenomena are inherent to large ensembles, their features are determined by characteristics which make physical sense also for unitary systems [6]. Indeed, synchronization (and clustering) in ensembles and their susceptibility to control are influenced by their individual robustness properties. "Reliability" [9] (or "consistency" [10]), i.e. the stability of the system response to noisy driving, and coherence 6 6111, i.e. the constancy of the oscillation frequency in time, are the principal ones.

Mathematically, the natural quantifier of reliability is the Lyapunov exponent $(\mathrm{LE}, \lambda)$ measuring the exponential decay rate of perturbations of the system response [6]12]13]. Oscillators are more reliable for a larger negative LE. Coherence can be quantified by the diffusion constant (DC, $D$ ) of the oscillation phase $\varphi(t)$, which grows non-uniformly in time due to either noise or chaotic dynamics; $\left\langle(\varphi(t)-\langle\dot{\varphi}\rangle t)^{2}\right\rangle \propto$

\footnotetext{
${ }^{a}$ e-mail: Denis.Goldobin@gmail.com
} 
$D t$. These quantifiers are immediately relevant for the synchronization phenomenon in ensembles of oscillators subject to common noise [15/14] or global coupling [5 16 17].

In this paper, we report how the reliability and coherence of a noisy limit-cycle oscillator can be efficiently controlled by a general linear feedback. However, the ratio of LE and DC is shown to be independent of the noise strength and feedback parameters. The persistence of this ratio can be formulated as an uncertainty principle. Finally, the important implications of this principle for controlling collective dynamics of ensembles are revealed - the ensemble responds to control essentially differently for the cases of identical oscillators with intrinsic noise and slightly non-identical oscillators with no intrinsic noise - and conclusions are drawn. The tentative hypothesis on the response of ensembles to the control proposed in the concluding remarks of 25] is shown to be valid only for the case of non-identical oscillators with no intrinsic noise.

\section{General linear feedback}

Let us consider a general $N$-dimensional limit-cycle oscillator subject to small linear delayed feedback and weak noise:

$$
\dot{x}_{i}=F_{i}(\mathbf{x})+a z_{i}(t)+B_{i}(\mathbf{x}) \circ \xi(t),
$$

where $i=1,2, \ldots, N, a$ is the feedback strength, the feedback term

$$
z_{i}(t)=\int_{0}^{+\infty} \sum_{j=1}^{N} G_{i j}\left(t_{1}\right) x_{j}\left(t-t_{1}\right) d t_{1},
$$

$G_{i j}(t)$ is the Green's function, different $G_{i j}(t)$ can feature linear "single" or recursive delay feedback (particular forms of this function are specified in the text below) 1118, linear frequency filter [22, etc. The sign "o" indicates the Stratonovich form of equation; $\xi(t)$ is white Gaussian noise: $\langle\xi\rangle=0,\left\langle\xi(t) \xi\left(t^{\prime}\right)\right\rangle=2 \delta\left(t-t^{\prime}\right) ; B_{i}(\mathbf{x})$ is the susceptibility of the system to noise driving.

In nature and technology, oscillations of systems meant to be periodic are never perfectly periodic; noise is always present even for clocks, lasers, electronic generators, etc. On the other hand, for the majority of systems of practical interest their natural dynamics is non-overwhelmed by noise. We typically deal with natural system dynamics affected by noise rather than with strong noise signal transformed by the system. Therefore, the case of weak noise can be treated as a typical one, and we restrict our consideration to this case. For weak noise and feedback the dynamics can be described within the framework of the phase description up to the leading order of accuracy 2324$]$ :

$$
\begin{gathered}
\dot{\varphi}=\Omega_{0}+a \int_{0}^{+\infty} \sum_{i=1}^{N} \sum_{j=1}^{N} G_{i j}\left(t_{1}\right) H_{i j}\left(\varphi\left(t-t_{1}\right), \varphi(t)\right) d t_{1} \\
+\varepsilon f(\varphi(t)) \circ \xi(t),
\end{gathered}
$$

where $\Omega_{0}$ is the natural frequency of the oscillator, $f$ is a $2 \pi$-periodic function featuring the sensitivity of the phase to noise, $\varepsilon$ is the noise amplitude, $H_{i j}(\psi, \varphi)$ is the increase of the phase growth rate created by the feedback term $x_{j}(\psi)$ acting on the variable $x_{i}(\varphi)$. One can calculate $H_{i j}(\psi, \varphi)=\left(\partial \varphi / \partial x_{i}\right) x_{j}(\psi)$ for a known phase field $\varphi(\mathbf{x})$ (see [20] and, e.g., 21] for examples of calculation of $\varphi(\mathbf{x})$; the Maple-program in supplementary material for [19] calculates $\left.H_{i j}(\psi, \varphi)\right)$. We would like to notice that 

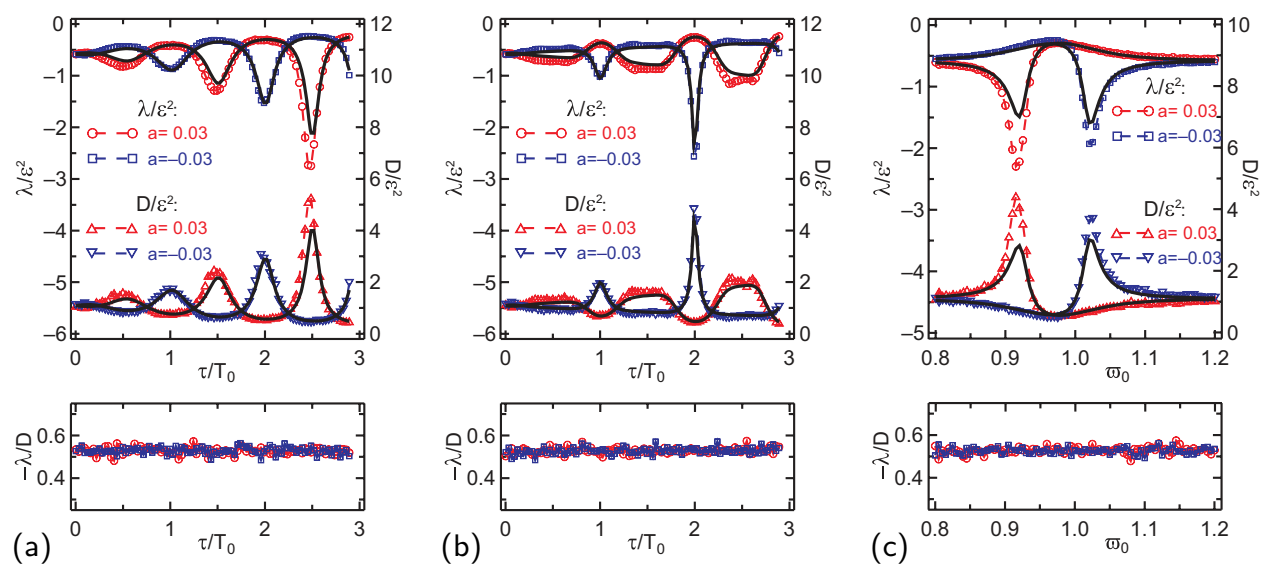

Fig. 1. (Color online) Dependencies of the Lyapunov exponent $\lambda$ and the diffusion constant $D$ (upper and lower graphs in the upper row of plots, respectively) on the feedback/filter parameters for the van der Pol oscillator with $\mu=0.7$ subject to Gaussian white noise of strength $\varepsilon=0.05$. (a) "Simple" delay feedback [eq. (8)]. (b) Recursive delay feedback [eq. (9)]. (c) Linear frequency filter [eq. (10)] with $\alpha=0.1$. The oscillation period of the control-free noiseless system $T_{0} \approx 2 \pi / 0.97$; the feedback strength $(a=0.03$ and $a=-0.03)$ is specified in plots. Solid black lines present analytical dependencies [eqs. (3) - (5)] with $\left\langle f^{2}\right\rangle \approx 0.5464$ and $\left\langle\left(f^{\prime}\right)^{2}\right\rangle \approx 0.5775$. The ratio between the Lyapunov exponent and diffusion constant is constant and obeys eq. (6) up to the calculation accuracy (see the lower row of plots).

eq. (2) is not an approximation but an accurate mathematical description for the case we consider.

The phase of the noise-free oscillator grows uniformly and its shifts neither decay nor grow in time. Noise creates irregularity of the phase growth rate, measured by the phase diffusion constant $D(\mathrm{DC}):\left\langle(\varphi(t)-\langle\varphi(t)\rangle)^{2}\right\rangle \propto D t$. Additionally, noise results in convergence of trajectories, phase shifts decay, and the exponential rate of this decay is measured by the Lyapunov exponent $\lambda(\mathrm{LE})$.

In 22 the analytical calculation of DC was extended to the general case of linear feedback, which covers the particular cases of simple and recursive delay feedback 111819 and was employed for the case of linear frequency filter. The derivation procedure for LE [25] is essentially more laborious than that for DC even for the case of simple delay feedback and is strictly limited to the case of weak noise (meanwhile the analytical evaluation of DC does not necessarily require noise to be weak [1]). Here we skip the derivation procedure due to its laboriousness and the fact that it relies on ideas previously elaborated [25], and provide the results.

For the general case of linear feedback, mean frequency $\Omega$, phase diffusion constant $D$, and Lyapunov exponent $\lambda$ read

$$
\begin{gathered}
\Omega=\Omega_{0}+a \int_{0}^{+\infty} \sum_{i=1}^{N} \sum_{j=1}^{N} G_{i j}(t) h_{i j}(-\Omega t) d t \\
D=\frac{2 \varepsilon^{2}\left\langle f^{2}\right\rangle_{\varphi}}{\left(1+a \int_{0}^{+\infty} t \sum_{i=1}^{N} \sum_{j=1}^{N} G_{i j}(t) h_{i j}^{\prime}(-\Omega t) d t\right)^{2}} \\
=2 \varepsilon^{2}\left\langle f^{2}\right\rangle_{\varphi}\left(\frac{\partial \Omega}{\partial \Omega_{0}}\right)^{2},
\end{gathered}
$$




$$
\begin{gathered}
\lambda=-\frac{\varepsilon^{2}\left\langle\left(f^{\prime}\right)^{2}\right\rangle_{\varphi}}{\left(1+a \int_{0}^{+\infty} t \sum_{i=1}^{N} \sum_{j=1}^{N} G_{i j}(t) h_{i j}^{\prime}(-\Omega t) d t\right)^{2}} \\
=-\varepsilon^{2}\left\langle\left(f^{\prime}\right)^{2}\right\rangle_{\varphi}\left(\frac{\partial \Omega}{\partial \Omega_{0}}\right)^{2},
\end{gathered}
$$

where $\langle\ldots\rangle_{\varphi} \equiv(2 \pi)^{-1} \int_{0}^{2 \pi} \ldots d \varphi, h_{i j}(\psi):=\left\langle H_{i j}(\varphi+\psi, \varphi)\right\rangle_{\varphi}$, and the prime denotes derivative. Notice, for any kind of linear feedback the ratio

$$
\frac{-\lambda}{D}=\frac{\left\langle\left(f^{\prime}\right)^{2}\right\rangle}{2\left\langle f^{2}\right\rangle}
$$

is independent of parameters of the feedback. Previously this ration was observed only for the case of simple delay feedback.

The modified formulation of the result (6) was revealed also for a certain class of systems where the phase cannot be well defined. Specifically, in systems below a Hopf bifurcation, noise can induce oscillatory motion 26 for which the phase is not well defined. For slightly perturbed periodic oscillations the autocorrelation function $C_{j j}(s):=\left\langle x_{j}(t) x_{j}(t+s)\right\rangle$ decays exponentially, $C_{j j}(s)=c_{j}(s) \exp (-D|s|)$, where function $c_{j}(s)$ oscillates but neither grows nor decays asymptotically. One can introduce an alternative quantifier of the irregularity of oscillations, the correlation time $t_{\text {corr }}:=C_{j j}^{-1}(0) \int_{0}^{\infty}\left|C_{j j}(s)\right| d s$ and find $t_{\text {corr }} \propto 1 / D$ for perturbed periodic oscillations. For noise-induced oscillations, $t_{\text {corr }}$ is an appropriate quantifier, while $D$ cannot be evaluated. In Refs. 26, for the van der Pol oscillator slightly below a Hopf bifurcation, the product $t_{\text {corr }}|\lambda|$ was reported to be independent of the feedback with time-delay(s), although the feedback significantly changed $t_{\text {corr }}$ and $\lambda$. This is equivalent to the result (6).

The validity of our findings (3)-(6) can be underpinned with the results of numerical simulation for noisy van der Pol oscillator

$$
\dot{x}=y, \quad \dot{y}=\mu\left(1-4 x^{2}\right) y-x+a z(t)+\varepsilon \xi(t) .
$$

Here $\mu$ describes closeness to the Hopf bifurcation point, the phase $\varphi=-\arctan (y / x)$ (for $\mu \ll 1$ the limit cycle is: $x=\cos \varphi, y=-\sin \varphi$ ). We consider 3 possible cases: (1) a "simple" delay feedback with delay time $\tau$;

$$
z^{(1 \tau)}(t)=2(y(t-\tau)-y(t))
$$

which yields

$$
\hat{\mathbf{G}}^{(1 \tau)}(t)=\left[\begin{array}{lc}
0 & 0 \\
0 & 2(\delta(t-\tau)-\delta(t-0))
\end{array}\right]
$$

(here we explicitly indicate that $\int_{0}^{+\infty} \delta(t-0) d t=1$ ).

(2) a recursive delay feedback;

$$
z^{(\mathrm{m} \tau)}(t)=2 \sum_{n=0}^{+\infty} R^{n}(y(t-(n+1) \tau)-y(t-n \tau))
$$

where $|R|<1$, which yields

$$
\begin{aligned}
& \hat{\mathbf{G}}^{(\mathrm{m} \tau)}(t) \\
& \quad=\left[\begin{array}{cc}
0 & 0 \\
02 \sum_{n=0}^{+\infty} R^{n}(\delta(t-(n+1) \tau)-\delta(t-n \tau-0))
\end{array}\right] .
\end{aligned}
$$



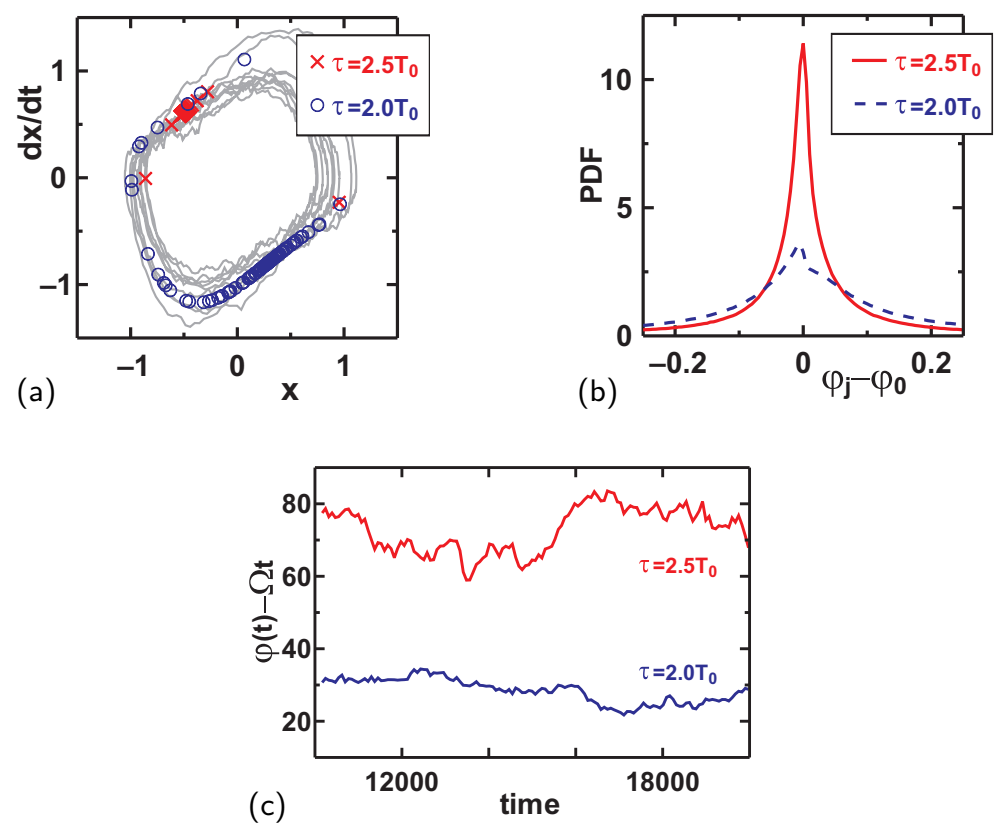

Fig. 2. (Color online) Ensemble of 100 van der Pol oscillators (7) with nonidentical frequencies subject to the simple delay feedback (8). The distribution of frequencies is the gaussian one centered at 1 with standard deviation 0.001 , feedback strength $a=0.03$, noise amplitude $\varepsilon=0.1$. (a) The gray line plots trajectory of one oscillator, the symbols plot snapshots of the ensemble for two specified values of the delay time (check these values in Fig. 1a). (b) The distribution of the phase deviations from the value $\varphi_{0}$ corresponding to the instantaneous ensemble-mean state. (c) The deviation of the phase from its mean growth for an oscillator (the offset of the vertical axis is arbitrary).

(3) a linear frequency filter;

$$
z^{(\mathrm{lff})}(t)=2 \dot{u}(t), \quad \ddot{u}+\alpha \dot{u}+\tilde{\omega}_{0}^{2} u=\alpha x(t),
$$

which yields

$$
\hat{\mathbf{G}}^{(\mathrm{lff})}(t)=\left[\begin{array}{cc}
0 & 0 \\
2 \alpha e^{-\alpha t / 2}\left(\cos \omega_{0} t-\frac{\alpha}{2 \omega_{0}} \sin \omega_{0} t\right) & 0
\end{array}\right],
$$

$\omega_{0}=\sqrt{\tilde{\omega}_{0}^{2}-(\alpha / 2)^{2}}$.

For $\mu \ll 1$, the van der Pol oscillator possesses a circular limit-cycle and $h_{21}(\varphi)=$ $-(1 / 2) \cos \varphi, h_{22}(\varphi)=(1 / 2) \sin \varphi, f(\varphi)=-\sin \varphi$. The analytical results derived with the above formulae match well the results of numerical simulation [11822, as can be well seen in Fig. 1. Remarkably, the ratio between LE and DC is constant with a good accuracy even when they vary by one order of magnitude, in agreement with eq. (6).

\section{Uncertainty principle}

Constancy of the ratio (6),

$$
\frac{-\lambda}{D}=\frac{\left\langle\left(f^{\prime}\right)^{2}\right\rangle}{2\left\langle f^{2}\right\rangle}
$$



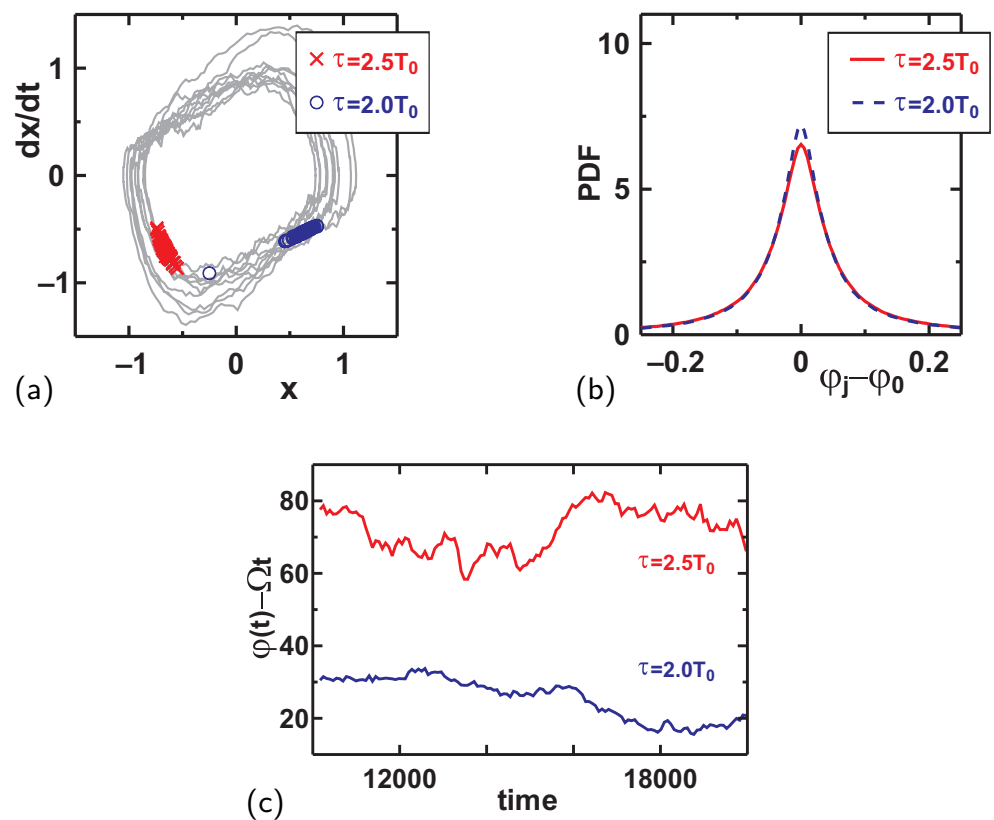

Fig. 3. (Color online) Ensemble of 100 van der Pol oscillators (7) subject to the simple delay feedback (8), common noise $\varepsilon=0.1$ and intrinsic noise $\varepsilon_{\text {int }}=0.005$. For details see caption to Fig. 2

can be formulated as a kind of uncertainty principle for the linear feedback control techniques:

The reliability of a noisy oscillator can be significantly enhanced (by means of a weak linear feedback) but at the price of the loss of its coherence and, vice versa, the coherence can be significantly enhanced but with the loss of reliability.

\section{Implications for ensembles of oscillators}

For ensembles of uncoupled oscillators driven by common noise we know, that the imperfectness of identity of oscillators or intrinsic noise leads to imperfectness of synchronization. The characteristic spreading of states $\propto 1 / \sqrt{-\lambda}[15$. That is, higher reliability leads to stronger synchrony of oscillations. Thinking of employment of control techniques we should distinguish two "pure" situations: (i) slightly non-identical oscillators driven by identical noise and (ii) identical oscillators with small intrinsic noise, individual for each oscillator.

For an ensemble of non-identical oscillators, the dispersing of phases is owed to the mismatch of natural frequencies. This mismatch is nearly unaffected by small feedback, while a synchronizing action measured by LE is influenced by the feedback. Hence, the dispersion of states is perceptive to the feedback control. For instance, one can see in Fig. 2 that the ensemble is well synchronized (Fig. 2a,b) when the phase diffusion is large (Fig. 2r), and, on the contrary, for a small diffusion (enhanced coherence) we observe a much poorer synchrony.

For an ensemble of identical oscillators with intrinsic noise, the situation is different. The dispersion is owed to the mutual diffusion of oscillator phases created by intrinsic noise. Similarly to [15, we can find $\Delta \varphi \propto \sqrt{D_{\text {int }}} / \sqrt{-\lambda}$, where $D_{\text {int }}$ characterizes the diffusion due to intrinsic noise. The diffusion owing to intrinsic noise 

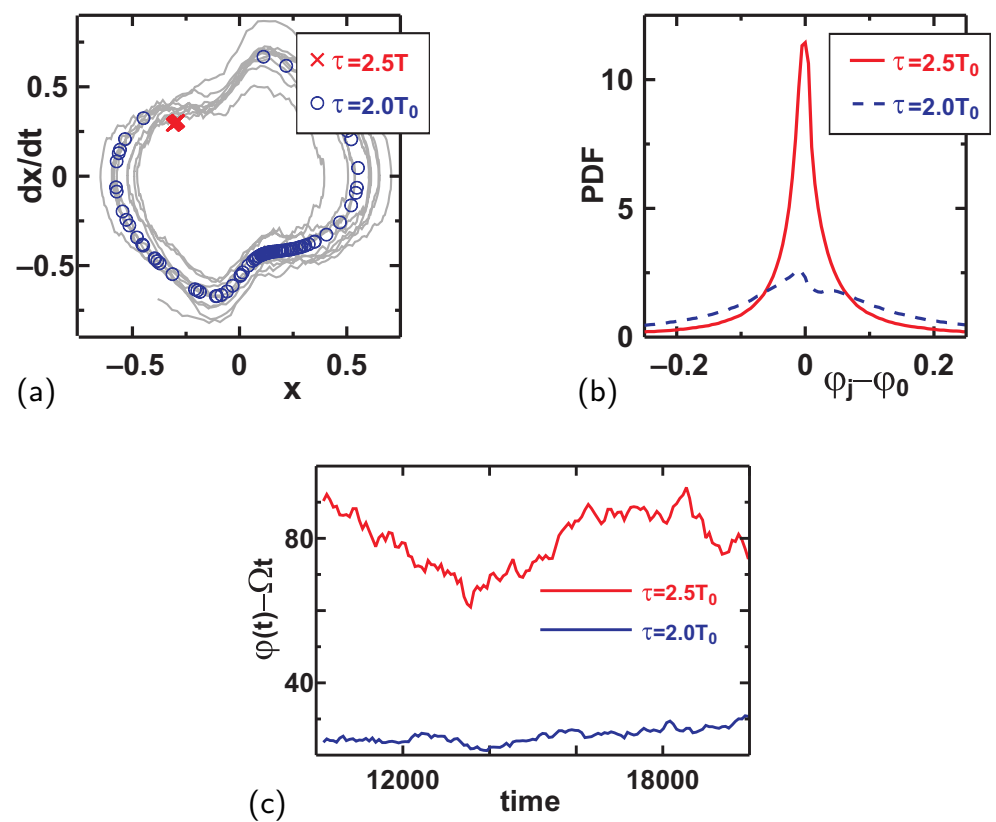

Fig. 4. (Color online) Ensemble of 100 pendulum clock oscillators (11) with $\mu=0.4$ and nonidentical frequencies subject to the simple delay feedback (8). The distribution of $\widetilde{\Omega}_{0}$ is the gaussian one centered at 1 with standard deviation 0.001 , feedback strength $a=0.03$, noise amplitude $\varepsilon=0.05$. For details see caption to Fig. 2 ,

is expected to be subjected to the effect of the feedback in the same manner as the total diffusion. Hence, we expect the feedback to not influence the phase dispersion, $\Delta \varphi \propto \sqrt{D_{\text {int }}} / \sqrt{-\lambda} \approx$ const. Indeed, in Fig. 3 one can see that the distribution of phase deviations is tolerant to feedback control. In this case, one can employ the feedback control techniques to control the coherence without the loss of synchrony.

Weakness of noise - which is the condition of accuracy of relation (6) - is the feature of clock systems. Simultaneously, the accuracy and synchronization effects are especially important for clock oscillators. Van del Pol oscillator may be treated as a rough approximation of the pendulum clock oscillator [27. A more appropriate model for the pendulum clock oscillator (with feedback control and noise) is

$$
\ddot{x}+\mu \dot{x}+\widetilde{\Omega}_{0}^{2} \sin x=\operatorname{sign}(\dot{x}) M(x)+a z(t)+\varepsilon \xi(t),
$$

where function $M(x)$ is localized near $x=0$ 27]. For demonstration we adopt $M(x)=$ $M_{0} x_{M}^{-1} \exp \left(-x^{2} / x_{M}^{2}\right)$ with $x_{M}=0.1$ and $M_{0}=0.1$. In Fig. 4. one can see that for the ensemble of nonidentical systems subject to common noise the synchrony is strongly influenced by the feedback control as well as the coherence, and they cannot be enhanced simultaneously. In Fig. [5. for identical oscillators with additional inherent noise synchrony is unaffected by feedback control, while the coherence can be significantly enhanced, as well as for Van der Pol oscillators.

The tentative hypothesis on the response of ensembles to the control proposed in the concluding remarks of Ref. 25] turned out to be valid only for the case of non-identical oscillators with no intrinsic noise. 

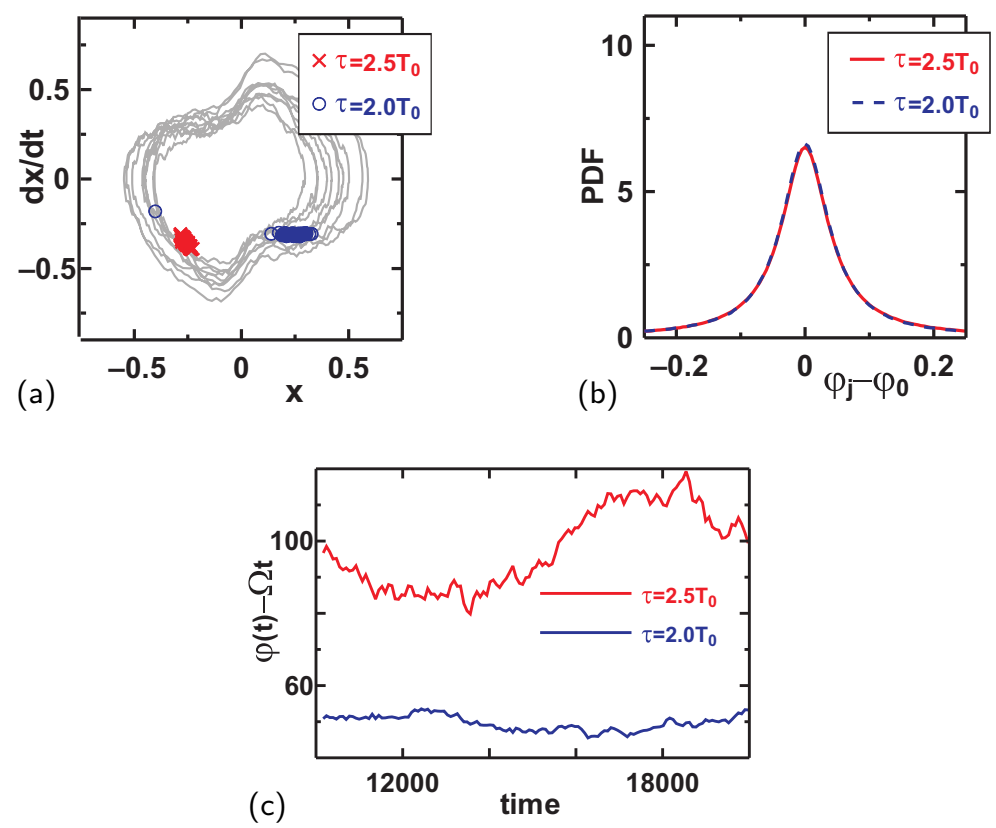

Fig. 5. (Color online) Ensemble of 100 pendulum clock oscillators (11) with $\mu=0.4$ subject to the simple delay feedback (8), common noise $\varepsilon=0.05$ and intrinsic noise $\varepsilon_{\text {int }}=0.0025$. For details see caption to Fig. 2,

\section{Conclusion}

Summarizing, we have discovered the universal uncertainty principle, which is valid for general noisy limit-cycle oscillators subject to a general linear feedback control and proved it both analytically and numerically. Mathematically, this uncertainty principle takes the form of eq. (6); the ratio of the Lyapunov exponent $(\lambda)$, measuring reliability (or the response stability, 910 ), and the diffusion constant $(D)$, measuring coherence (or the constancy of the instantaneous frequency in time), is independent of the noise strength and feedback parameters. That is the reliability of a noisy oscillator can be significantly enhanced by means of a relatively weak linear feedback, but at the price of the loss of its coherence, and vice versa, the coherence can be significantly enhanced but with the loss of reliability. The principle has an implication to practical issues of the control of synchronization in non-ideal ensembles of oscillators in nature and technology [1/2 46 14 17]. For ensembles of weakly non-identical oscillators driven by common noise (cf Fig. 2) the enhancement of synchrony, achieved due to the reliability increase, results in a poorer coherence of each individual oscillator. Unsimilarly, for ensembles of identical oscillators with intrinsic noise (cf Fig. 3) the synchrony is not influenced by the change of reliability/coherence; therefore, coherence can be controlled without stray effects on the synchronization.

Notice, that ensembles of globally coupled oscillators in an asynchronous state immediately correspond to the case of uncoupled oscillators receiving common driving; this driving is the ensemble-mean value forcing an oscillator via the coupling term [16]. As long as collective modes vanish, the ensemble-mean value fluctuating about zero may be well considered as a stochastic process and one can speak of synchronization by common noise. (When the collective mode appears, it can be regular in time; synchronization by a regular signal drastically differs from that by a nonperiodic one, 
e.g. 1328 .) Therefore, our findings are related to the fundamentals of synchronization in ensembles of globally coupled oscillators as well [17/29].

\section{References}

1. T. Danino, O. Mondragon-Palomino, L. Tsimring and J. Hasty, A synchronized quorum of genetic clocks, Nature 463, 326 (2010).

2. B. T. Grenfell et al., Noise and determinism in synchronized sheep dynamics, Nature 394, 674 (1998).

3. T. Kohonen, Self-Organizing Maps (Springer, Heidelberg, 2001) 3rd ed.

4. P. A. Tass, Phase Resetting in Medicine and Biology, Stochastic Modelling and Data Analysis (Springer-Verlag, Berlin, 1999).

5. S. H. Strogatz et al., Theoretical mechanics: Crowd synchrony on the Millennium Bridge, Nature 438, 43 (2005).

6. A. Pikovsky, M. Rosenblum and J. Kurths, Synchronization: A Universal Concept in Nonlinear Sciences (Cambridge University Press, Cambridge, 2001, 2003).

7. Ch. Zhou, J. Kurths, I. Z. Kiss and J. L. Hudson, Noise-Enhanced Phase Synchronization of Chaotic Oscillators, Phys. Rev. Lett. 89, 014101 (2002).

8. S. Haykin, Neural Networks and Learning Machines (Prentice Hall, 2008) 3rd ed.

9. Z. F. Mainen and T. J. Sejnowski, Reliability of Spike Timing in Neocortical Neurons, Science 268, 1503 (1995).

10. A. Uchida, R. McAllister and R. Roy, Consistency of nonlinear system response to complex drive signals, Phys. Rev. Lett. 93, 244102 (2004).

11. D. Goldobin, M. Rosenblum and A. Pikovsky, Controlling oscillator coherence by delayed feedback, Phys. Rev. E 67, 061119 (2003); Coherence of noisy oscillators with delayed feedback, Physica A 327, 124 (2003).

12. A. S. Pikovsky, Synchronization and stochastization of the ensemble of autogenerators by external noise, Radiophys. Quantum Electron. 27, 576 (1984); J. N. Teramae and D. Tanaka, Robustness of the Noise-Induced Phase Synchronization in a General Class of Limit Cycle Oscillators, Phys. Rev. Lett. 93, 204103 (2004); K. Pakdaman and D. Mestivier, Noise induced synchronization in a neuronal oscillator, Physica D 192, 123 (2004); D. S. Goldobin and A. S. Pikovsky, Synchronization of self-sustained oscillators by common white noise, Physica A 351, 126 (2005).

13. J. Ritt, Evaluation of entrainment of a nonlinear neural oscillator to white noise, Phys. Rev. E 68, 041915 (2003).

14. H. Nakao, K. Arai and Y. Kawamura, Noise-Induced Synchronization and Clustering in Ensembles of Uncoupled Limit-Cycle Oscillators, Phys. Rev. Lett. 98, 184101 (2007); K. Yoshida, K. Sato and A. Sugamata, Noise-induced synchronization of uncoupled nonlinear systems, J. Sound Vibration 290, 34 (2006); R. F. Galan, G. B. Ermentrout and N. N. Urban, Stochastic dynamics of uncoupled neural oscillators: Fokker-Planck studies with the finite element method, Phys. Rev. E 76, 056110 (2007); T. Zhou, J. Zhang, Zh. Yuan and L. Chen, Synchronization of genetic oscillators, Chaos 18, 037126 (2008).

15. D. S. Goldobin and A. Pikovsky, Synchronization and desynchronization of self-sustained oscillators by common noise, Phys. Rev. E 71, 045201(R) (2005).

16. D. Topaj, W.-H. Kye and A. Pikovsky, Transition to Coherence in Populations of Coupled Chaotic Oscillators: A Linear Response Approach, Phys. Rev. Lett. 87, 074101 (2001).

17. M. G. Rosenblum and A. S. Pikovsky, Controlling synchronization in an ensemble of globally coupled oscillators, Phys. Rev. Lett. 92, 114102 (2004); D. S. Goldobin and A. Pikovsky, Effects of Delaed Feedback on Kuramoto Transition, Prog. Theor. Phys. Suppl. 161, 43 (2006); O. V. Popovych, C. Hauptmann and P. A. Tass, Control of neuronal synchrony by nonlinear delayed feedback, Biol. Cybern. 95, 69 (2006).

18. A. H. Pawlik and A. Pikovsky, Control of oscillators coherence by multiple delayd feedback, Phys. Lett. A 358, 181 (2006). 
19. D. S. Goldobin, Anharmonic resonances with recursive delay feedback, Phys. Lett. A 375, 3410 (2011).

20. J. Guckenheimer, Isochrons and phaseless sets, J. Math. Biol. 1, 259-273 (1975).

21. K. Arai and H. Nakao, Phase coherence in an ensemble of uncoupled limit-cycle oscillators receiving common Poisson impulses, Phys. Rev. E 77, 036218 (2008).

22. N. Tukhlina, M. Rosenblum and A. Pikovsky, Controlling coherence of noisy and chaotic oscillators by a linear feedback, Physica A 387, 6045 (2008).

23. Y. Kuramoto, Chemical Oscillations, Waves and Turbulence (Dover, New York, 2003).

24. D. S. Goldobin, J.-N. Teramae, H. Nakao and G. B. Ermentrout, Dynamics of LimitCycle Oscillator Subject to General Noise, Phys. Rev. Lett. 105, 154101 (2010).

25. D. S. Goldobin, Coherence vs. Reliability of Stochastic Oscillators with Delayed Feedback, Phys. Rev. E 78, 060104(R) (2008).

26. N. B. Janson, A. G. Balanov and E. Schöll, Delayed Feedback as a Means of Control of Noise-Induced Motion, Phys. Rev. Lett. 93, 010601 (2004); J. Pomplun, A. Amann and E. Schöll, Mean-field approximation of time-delayed feedback control of noise-induced oscillations in the Van der Pol system, Europhys. Lett. 71(3), 366 (2005); J. Pomplun, A. G. Balanov and E. Schöll, Long-term correlations in stochastic systems with extended time-delayed feedback, Phys. Rev. E 75, 040101(R) (2007).

27. M. Kapitaniak, K. Czolczynski, P. Perlikowski, A. Stefanski and T. Kapitaniak, Synchronization of clocks, Phys. Rep. 517(12), 1-69 (2012).

28. D. S. Goldobin, Synchronization of Limit Circle Oscillators by Telegraph Noise, in Unresolved Problems and Fluctuations: UPoN 2005, edited by L. Reggiani et al., AIP Conf. Proc. 800, 394 (2005).

29. A. E. Hramov, A. A. Koronovskii and O. I. Moskalenko, Generalized synchronization onset, Europhys. Lett. 72, 901 (2005); Are generalized synchronization and noise-induced synchronization identical types of synchronous behavior of chaotic oscillators?, Phys. Lett. A 354, 423 (2006). 